\title{
Iron Ears: primeiras impressões de um jogo educativo para ensino de estrutura de dados lineares
}

\author{
Alexandre da Rosa ${ }^{1}$, Andreia de Jesus ${ }^{1}$, Gabriel Vaz Igarashi ${ }^{1}$, Vinicius Struginski Pereira ${ }^{1}$ \\ ${ }^{1}$ Setor de Educação Profissional e Tecnológica - Universidade Federal do Paraná (UFPR) \\ Curitiba - PR - Brazil \\ \{alexandrerosa, andreia.jesus, gabriel.igarashi,struginskipereira\}@ufpr.br
}

\begin{abstract}
Due to the technological evolution, different kinds of educational software have been gaining even more space in classrooms. Among them, there are the educational games, which promote both entertainment and ludic learning. Considering the struggle students from computing areas have to learn data structures, the game Iron Ears: Data Structure was chosen to be used in the course Data Structures 1 in the college Program of Technology in Analysis and Systems Development at Federal University of Paraná (UFPR). After carrying out experiments, it was possible to notice Iron Ears helped in student retention and it helped to improve their understanding of the content, this conclusion was based on final scores achieved by students and their attendance.
\end{abstract}

Resumo. Com a evolução tecnológica os softwares educacionais ganham cada vez mais espaço em sala de aula. Dentre estes, existem os jogos educativos, que promovem tanto o entretenimento quanto o ensino através do lúdico. Levando em conta a dificuldade dos alunos da área de computação na aprendizagem de estruturas de dados, optou-se pela aplicação do jogo Iron Ears: Data Structure na disciplina de Estruturas de Dados I do curso superior de Tecnologia em Análise e Desenvolvimento de Sistemas da Universidade Federal do Paraná (UFPR). A partir dos experimentos realizados, com base nas médias finais e frequência dos alunos, foi possível notar que o Iron Ears ajudou na retenção de alunos e melhorou a compreensão do conteúdo dos mesmos.

\section{Introdução}

O tratamento e armazenamento de informações é o cerne do desenvolvimento de sistemas, assim sendo, como [Lawrence 2004] ressalta, o bom conhecimento de Estruturas de Dados e sua manipulação é um dos pilares para qualquer estudante na área de Tecnologia da Informação. No entanto, por ser algo abstrato, a assimilação de seus conceitos e sua utilização adequada podem ser tarefas árduas. Esta concepção é um fato comum na visão de diversos professores da área, como indicam as notas finais e a taxa de desistência dos alunos tanto na disciplina de Estruturas de Dados quanto em outras que possuam conteúdos introdutórios a mesma [Vieira et al. 2015].

Com o intuito de auxiliar discentes do curso superior de Tecnologia em Análise e Desenvolvimento de Sistemas (TADS) da Universidade Federal do Paraná (UFPR) no aprendizado deste conteúdo, em 2017 foi desenvolvido o Iron Ears: Data Structure, um jogo educativo que tem o objetivo de abranger de forma implícita e lúdica o funcionamento e a aplicação de algumas estruturas de dados [da Rosa et al. 2018]. 
Portanto, o presente artigo apresenta o jogo educativo desenvolvido, demonstrando o método de avaliação, assim como os resultados obtidos no uso do Iron Ears como ferramenta de auxílio no ensino-aprendizagem de Estrutura de Dados Lineares, além de sua contribuição no aumento do engajamento dos alunos na disciplina nesta avaliação piloto, fornecendo dados para embasar avaliações mais específicas futuramente.

\section{Referencial Teórico}

Considerando as dificuldades que aulas unicamente expositivas possuem em transmitir os conhecimentos sobre estrutura de dados, procurou-se alternativas distintas, que fizessem uso de novos recursos disponíveis. Dentre estes, um que se destaca quando se trata de métodos de ensino são os softwares educacionais e educativos, os quais possuem o intuito de auxiliar no processo pedagógico.

Devido a sua variedade de abordagens e soluções, os softwares educacionais posuem diversas categorias, tais como: Tutoriais; Exercício e Prática; Programação; Aplicativos; Multimídia e Internet; Simulação e Modelagem; Jogos [Vieira 2001].

Dentre as opções disponíveis em tal classificação optou-se pelos jogos educativos, que são muito eficientes quando se trata de ensinar, pois enquanto o conhecimento é transmitido, eles divertem, motivam e desafiam, aumentando o desenvolvimento de capacidades intelectuais e a absorção do conteúdo do jogador [Tarouco et al. 2004].

Os jogos educativos são meios relevantes de apresentar conteúdos que o processo de ensino em sala de aula não é capaz de transmitir em sua totalidade. [Van Eck 2006] afirma que os jogos possuem uma grande eficácia, por relacionarem diretamente o que se deve aprender com o contexto apresentado no mesmo, já que desta forma o aprendizado não é somente algo pertinente, mas também possui aplicação prática neste ambiente. A sensação de aplicabilidade, unida com um contexto adequado dá uma capacidade única aos jogos educativos, assim como a solução aqui apresentada, trazendo uma nova perspectiva aos alunos sobre estrutura de dados.

Ainda assim, há uma grande dificuldade em comprovar empiricamente a eficácia dos jogos educativos. A dificuldade em se estabelecer quais são os elementos essenciais do software e quais são impactantes, muito devido aos diferentes gêneros de jogos, além do método de aplicação de cada ferramenta no processo de ensino-aprendizagem, agravam a dificuldade na obtenção de respostas contundentes [Connolly et al. 2012].

Mesmo com esta dificuldade, um número relevante de soluções no âmbito de jogos possui indicativos de melhora do desempenho dos alunos em suas respectivas disciplinas alvo. Alguns exemplos que indicam sua relevância no processo de ensinoaprendizagem são, o Sortia, que aborda estrutura de dados ensinando algoritmos de ordenação [Battistella et al. 2012], o DEG4Trees um jogo educativo para o ensino de estruturas de dados do tipo árvore [Barbosa et al. 2014] e o jogo desenvolvido por [Thiry et al. 2010] para o auxílio na disciplina de engenharia de requisitos.

\section{Sobre o Iron Ears}

Iron Ears: Data Structure ${ }^{1}$ é um jogo de quebra-cabeça e simulação em 2D, disponível nos idiomas português e inglês. Nele o jogador será desafiado a ajudar na gestão de linhas

\footnotetext{
${ }^{1}$ Versão para demonstração disponível em https://npc42-games.itch.io/ironears
} 
de produção de robôs. Quanto a jogabilidade, o controle do jogador consiste em movimentos de arrastar, utilizando o mouse, e soltar 'comandos' para a planilha de execução. Além de preenchimentos de campos específicos a partir do teclado e seleção de opções a partir de escolhas drop-down. Atualmente o jogo possui 20 fases, que exploram as Estruturas de Dados Lineares, onde o jogador deverá utilizar de seus conhecimentos para criar diversas destas estruturas, representadas por estações de trabalho, ao mover comandos referentes às operações inerentes a elas.

O objetivo principal do Iron Ears é possibilitar que o ensino-aprendizagem de Estruturas de Dados possa ser descomplicado e, mais que isso, servir como um incentivo para o seu aprendizado, fazendo uso do lúdico. A ideia não é a de remover a necessidade de se ter um responsável por ensinar ou instruir sobre os princípios básicos das estruturas de dados, mas pelo contrário, o Iron Ears surge como uma ferramenta a ser utilizada em conjunto com outras formas de ensino de Estrutura de Dados.

Portanto, as atividades ministradas com o Iron Ears foram feitas em sala de aula (laboratório de informática) antes da apresentação dos conceitos teóricos da disciplina, de forma a validar os conceitos construtivistas no qual o jogo é embasado, nas aulas de Estruturas de Dados I do curso TADS da UFPR. O objetivo foi que, a posteriori, os discentes conseguissem, por si só, relacionar os conteúdos do jogo com os conteúdos de estruturas de dados apresentados em a aula e, consequentemente, tirando-os do ponto de vista abstrato para um mais concreto, assim facilitando o entendimento do conteúdo.

\section{Resultados}

Os experimentos foram realizados durante o ano de 2019 com alunos da disciplina de Estrutura de Dados I do $3^{\circ}$ período do curso superior de Tecnologia em Análise e Desenvolvimento de Sistemas da Universidade Federal do Paraná.

Os alunos desta disciplina, no período de matrículas, foram separados de forma aleatória em duas turmas pela coordenação do curso. A primeira delas, a qual chamaremos de Grupo de Controle (GC) formada por 37 alunos. O GC não fez uso do Iron Ears: Data Structure. A segunda turma, que rotularemos como Grupo de Experimento (GE) composta por 29 alunos, fez uso do Iron Ears: Data Structure. Ambos os grupos passaram pelas mesmas avaliações e com condições iguais para aprovação, as quais são as seguintes: (1) alunos com frequência mínima de $75 \%$ das aulas e média igual ou superior a 7 são aprovados; (2) alunos com frequência mínima de $75 \%$ das aulas e média inferior a 7 e superior a 4, necessitam realizar exame final, sendo que após este, a média para aprovação é de igual ou superior a 5; (3) alunos com frequência inferior a 75\% das aulas são reprovados automaticamente.

Em busca de indicativos que o Iron Ears é capaz de incentivar os alunos a comparecerem nas aulas foi avaliado quantos alunos reprovaram por falta, ou seja, alunos que não compareceram a pelo menos $75 \%$ das aulas. No GC, que continha 37 alunos, dezesseis (16) alunos possuiu 16 alunos reprovaram por falta, aproximadamente $\mathbf{4 3 . 2 4 \%}$ da turma. Já o GE com 29 alunos, um (1) aluno, possuiu 1 aluno reprovou por falta, computando $3.44 \%$ de sua totalidade.

Para verificar se o Iron Ears indica impacto no aprendizado dos alunos foram avaliadas as notas finais na disciplina de ambos os grupos, desconsiderando os alunos 
reprovados por falta. A média das notas finais do GC foi 5.17, enquanto a média dos alunos do GE foi de 6.08. Com isto definem-se tais hipóteses: (H0): As notas finais do GC e do GE têm desempenhos semelhantes. (H1): As notas finais do GC e do GE têm desempenhos diferentes.

Através da aplicação do teste Wilcoxon-Mann-Whitney para amostras independentes [Siegel and Castellan Jr 1975], que avalia se os resultados da amostra das medianas das notas dos dois grupos são idênticas, os seguintes resultados foram obtidos:

$$
\mathrm{W}_{\mathrm{GC}}=422.500, \mathrm{~W}_{\mathrm{GE}}=802.500, \mathrm{p} \text {-valor-atribuído }=0.038
$$

Inicialmente observando-se a soma dos postos (W) de GC e a soma de GE, nota-se uma diferença considerável entre ambos os valores. Considerando o previamente estabelecido nível de significância de 5\%, rejeita-se a hipótese (H0), já que $p<0.05$, pois há menos de $5 \%$ de chance que a hipótese $\mathrm{H} 1$ esteja incorreta. Com isto, é possível afirmar que existe diferença significativa entre as notas finais dos alunos dos grupos GC e GE. A Figura 1 demonstra visualmente o espalhamento das notas obtidas nos testes finais de cada grupo, com uma melhora visível nas notas em geral do GE, assim como a diferença entre as medianas constata pelo teste de Wilcoxon-Mann-Whitney.

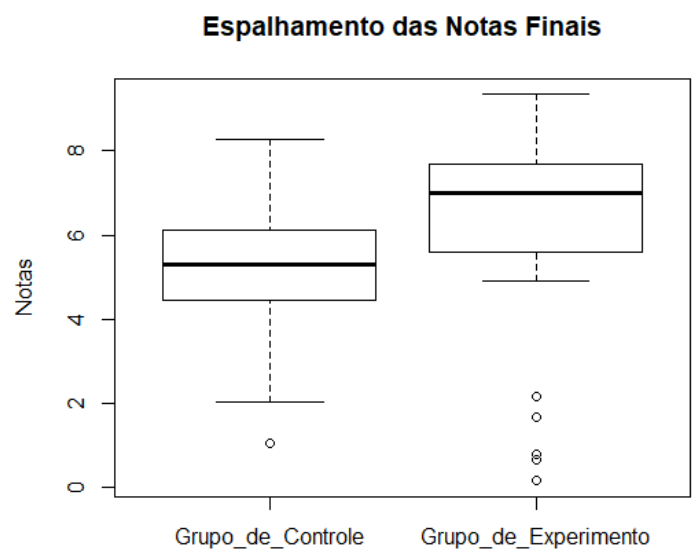

Figura 1. Espalhamento das notas representado em um diagrama de caixa

\section{Conclusão}

As dificuldades inerentes ao conteúdo de Estrutura de Dados instigam o desenvolvimento de meios educativos alternativos para se transmitir esse conteúdo. Neste artigo, apresentou-se o Iron Ears: Data Structure, um jogo educativo que se propõe a melhorar o processo de ensino-aprendizagem por meio do lúdico. Os experimentos realizados com as duas turmas de Estruturas de Dados I, por meio de um Grupo de Controle e outro Grupo de Experimento, apresentaram resultados significativos. Tanto os números de reprovação por falta quanto as notas finais dos estudantes indicam que o uso do jogo em sala de aula tem um impacto positivo no processo de ensino-aprendizagem.

Todavia, a coleta de dados com um número reduzido de alunos, atrelado a apontamentos de necessidade de melhora na usabilidade do jogo, levantada pelos alunos ao 
longo do experimento, evidencia a necessidade de mais testes. Portanto, com os dados obtidos e as sugestões de melhora, uma versão mais atualizada do jogo está sendo desenvolvida e novas turmas estão sendo preparadas para agora não só avaliar o impacto do jogo, mas o quão assertivo ele é nos aspectos que dizem respeito a um jogo educativo e a sua contribuição no processo de ensino-aprendizagem de Estruturas de Dados Lineares.

\section{Referências}

Barbosa, W. A., de Freitas Nunes, I., and Gondim, A. C. (2014). Deg4trees: Um jogo educacional digital de apoio ao ensino de estruturas de dados. Monografia de Graduação. Ciência da Computação. UFG/Regional Jataí.

Battistella, P. E., von Wangenheim, A., and von Wangenheim, C. G. (2012). Sortia-um jogo para ensino de algoritmo de ordenação: estudo de caso na disciplina de estrutura de dados. In Brazilian Symposium on Computers in Education (Simpósio Brasileiro de Informática na Educação-SBIE), volume 23.

Connolly, T. M., Boyle, E. A., MacArthur, E., Hainey, T., and Boyle, J. M. (2012). A systematic literature review of empirical evidence on computer games and serious games. Computers \& education, 59(2):661-686.

da Rosa, A., Igarashi, G. V., de Oliveira, R. H., Viana Filho, R., Pereira, V. S., and de Jesus, A. (2018). Iron ears: Educational game for teaching and learning of data structure.

Lawrence, R. (2004). Teaching data structures using competitive games. IEEE Transactions on Education, 47(4):459-466.

Siegel, S. and Castellan Jr, N. J. (1975). Estatística não-paramétrica para ciências do comportamento. Artmed Editora.

Tarouco, L. M. R., Roland, L. C., Fabre, M.-C. J. M., and Konrath, M. L. P. (2004). Jogos educacionais. RENOTE: revista novas tecnologias na educação [recurso eletrônico]. Porto Alegre, RS.

Thiry, M., Zoucas, A., and Gonçalves, R. Q. (2010). Promovendo a aprendizagem de engenharia de requisitos de software através de um jogo educativo. In Brazilian Symposium on Computers in Education (Simpósio Brasileiro de Informática na EducaçãoSBIE), volume 1.

Van Eck, R. (2006). Digital game-based learning: It's not just the digital natives who are restless. EDUCAUSE review, 41(2):16.

Vieira, C. E. C., de Lima Junior, J. A. T., and de Paula Vieira, P. (2015). Dificuldades no processo de aprendizagem de algoritmos: uma análise dos resultados na disciplina de al1 do curso de sistemas de informação da faeterj-campus paracambi. Cadernos UniFOA, 10(27):5-15.

Vieira, F. M. S. (2001). Avaliação de software educativo: reflexões para uma análise criteriosa. https://s3.amazonaws.com/academia.edu.documents/ $32016960 /$ Avaliacao_de_Software_Educativo_Reflexoes_para_ uma_Analise_Criteriosa.pdf. Acesso em 12 de março de 2020. 